PAPER

\title{
Intravenous rt-PA for acute stroke: comparing its effectiveness in younger and older patients
}

\author{
M S Mouradian, A Senthilselvan, G Jickling, J A McCombe, D J Emery, N Dean, A Shuaib
}

J Neurol Neurosurg Psychiatry 2005;76:1234-1237. doi: 10.1136/jnnp.2004.047803

See end of article for authors' affiliations

.....................

Correspondence to:

Dr A Shuaib, Department of Medicine, Division of Neurology, University of Alberta, 2E3.13 Walter C Mackenzie Health Sciences Centre, Edmonton,

Alberta, Canada T6G 2B7;

ashfaq.shuaib@

valberta.ca

Received 16 June 2004

In revised form

8 January 2005

Accepted 10 January 2005

\begin{abstract}
Objective: To study the short and long term differences in outcome between patients $\geqslant 80$ years of age and those $\leqslant 79$ years of age who received intravenous recombinant tissue plasminogen activator (iv rt-PA) for acute stroke within the first 3 hours of symptom onset.

Methods: We studied consecutive patients treated with iv rt-PA for acute stroke, with prospective follow up of up to 3 years. Outcome measures included National Institutes of Health Stroke Scale (NIHSS) score, Barthel Index (BI), modified Rankin score (MRS), and stroke mortality. Patients were split into two groups: younger ( $\leqslant 79$ years) and older ( $\geqslant 80$ years).

Results: There were 65 patients in the younger cohort and 31 patients in the older. Older patients were more likely to present with more severe baseline stroke ( $p=0.04$; odds ratio (OR) 3.04 ; $95 \%$ confidence interval (CI) 1.03 to 8.98 ). Stroke mortality at 90 days was $10.8 \%$ in the younger and $32.3 \%$ in the older cohort $(p=0.01)$. At 90 days' follow up, patients in the older cohort with more severe stroke (NIHSS score $\geqslant 11$ ) were nearly 10 times more likely to have poor outcome compared with their younger counterparts presenting with severe stroke $(p=0.001 ; O R=10.36 ; 95 \% \mathrm{Cl} 2.16$ to 49.20$)$. Baseline stroke severity and age were the only independent and equal predictors for stroke outcome. No threshold was found for age or baseline stroke severity predicting outcome.

Conclusion: Older patients presenting with more severe baseline stroke are much less likely to benefit from iv rt-PA as compared with their younger counterparts.
\end{abstract}

$\mathrm{F}$ ollowing the National Institute of Neurological Disorders and Stroke (NINDS) rt-PA Stroke Study, intravenous recombinant tissue plasminogen activator (iv rt-PA) was licensed in the USA and Canada for acute stroke within 3 hours of onset. ${ }^{1}$ The drug was later approved in Germany and by the European Agency for the Evaluation of Medical Products for the same time window. The NINDS trial demonstrated $11-14 \%$ absolute and nearly 30\% relative difference in excellent outcome favouring the treatment group at 90 days. This benefit was achieved despite 10 times increased risk of symptomatic haemorrhage in the treatment group, with no significant difference in mortality, as compared with controls. ${ }^{1}$ The benefit was sustained at 12 months. $^{2}$

While in France iv rt-PA is not indicated for patients above 80 years of age, licensing of iv rt-PA in Canada and USA indicates no upper age limit. However in the NINDS trial, only 42 patients were 80 years of age or older. ${ }^{3}$ A subgroup analysis of the NINDS trial data indicated that older age and higher baseline NIHSS score predicted less favourable outcome. ${ }^{4}$ While it was stated that there was no interaction between treatment and the variable expressed as age NIHSS score on multiple outcomes, indicating that age NIHSS score coupling "influenced outcome independently of treatment", the authors concluded that in the older group (166 patients aged $>75$ years were randomised), iv rt-PA offered mild to moderate benefit. ${ }^{4}$ In the NINDS trial, older patients with more severe stroke were more likely to do poorly. Thrombolytic therapy offered some benefit over placebo to this subgroup of patients, but the difference was not statistically significant. The authors indicated the need for further studies in older patients presenting with more severe stroke who are candidates for iv rt-PA. ${ }^{4}$ A 1 year follow up of patients in the NINDS trial reaffirmed that older patients or those with higher baseline NIHSS score were less likely to have favourable outcome. ${ }^{2}$ In a phase IV study, predictors of favourable outcome included baseline NIHSS score of $\leqslant 10$ and age $\leqslant 85$ years. $^{5}$ A retrospective study found no significant difference in attaining independent living at time of discharge from hospital, or in hospital mortality between patients of 80 years of age or older and their younger counterparts after receiving iv rt-PA. ${ }^{6}$ Although none of the aforementioned studies found a threshold age or stroke severity predicting outcome, to date no explanation has been offered to explain this phenomenon. A recent study found baseline stroke severity and age to be predictors of discharge to nursing home following acute stroke treated with iv rt-PA. ${ }^{7}$

In this paper, we report the results of our prospective study assessing the predictors of clinical outcome in two cohorts of patients, those of age $\leqslant 79$ years and those $\geqslant 80$ years who received iv rt-PA for acute stroke within 3 hours of symptom onset.

\section{METHODS}

From January 1999 to December 2001, all 96 consecutive patients who received iv rt-PA for acute stroke were included in this study. Patient demographics, past medical history (including history of cerebrovascular events, coronary artery disease, atrial fibrillation, congestive heart failure, valvular disease, hypertension, hyperlipidaemia, diabetes, smoking, use of antiplatelets), pre-stroke BI and MRS, temporal aspects of stroke onset to treatment, baseline NIHSS score, admission blood pressure, and blood glucose were recorded. Findings on baseline and follow up brain computed tomography (CT) scans were interpreted by neuroradiologists blinded to clinical presentation and outcome. Indication for iv rt-PA followed the guidelines suggested by the Canadian

Abbreviations: $\mathrm{BI}$, Barthel Index; $\mathrm{CT}$, computed tomography; iv rt-PA, intravenous recombinant tissue plasminogen activator; MRS, modified Rankin score; NIHSS, National Institutes of Health Stroke Scale; NINDS, National Institute of Neurological Disorders and Stroke 
Stroke Consortium. ${ }^{8}$ In essence these are similar to those of the American Heart Association recommendations. ${ }^{9}$ In accordance with Canadian guidelines, patients with CT evidence of clearly visible infarction involving more than one third of middle cerebral artery territory were not treated with iv rt-PA.

Follow up brain CT was obtained within 24 to 72 hours of treatment. Follow up NIHSS score, ${ }^{10} \mathrm{MRS}^{11}$ and $\mathrm{BI}^{12}$ during hospital stay, and at 3, 12, 24, 36, and 72 months were recorded. Patients were examined in their most current habitation (own home, rehabilitation, long term care facility). Follow up evaluation involved two physicians, (when necessary, the caregiver as well), and consensus had to be reached on BI and MRS. Cause of death was identified from hospital charts and/or from autopsy reports. Deaths caused by index stroke or its complications, were considered stroke related deaths. Deaths unrelated to stroke or its complications were considered incidental deaths. Symptomatic intracranial hemorrhage was defined as clinical deterioration with four points or more increase in the NIHSS score in the presence of intracranial haemorrhage that involved $>30 \%$ area of the stroke volume..$^{13-15}$

\section{Statistical analysis}

The distribution of continuous variables was described with means and standard deviations. Frequencies and percentages were used to describe nominal variables. The $\chi^{2}$ test and odds ratio (OR) with $95 \%$ confidence interval (CI) were used to determine the significance and strength of association between dichotomous variables. If the expected values were $<5$ in $2 \times 2$ tables, Fisher's exact test was used instead of $\chi^{2}$. Two independent sample $t$ tests were used to determine the significance of the difference in means between two groups. If the distribution was not normal or variances were not equal, the Mann-Whitney $U$ test was used instead of the $t$ test. Kaplan-Meier curves were used to describe the survival distribution of the younger and older age cohorts. The significant difference in the survival distribution was determined using the log-rank test. Multivariate logistic regression was used to determine independent predictor of stroke severity at 90 day follow up.

\section{RESULTS}

From January 1999 to December 2001, 96 patients received iv rt-PA for acute stroke within 3 hours of stroke onset. Of those, 65 patients were $\leqslant 79$ years of age (younger cohort, age range 32 to 79 ), and 31 were $\geqslant 80$ years of age (older cohort, age range 80 to 96 ). The younger cohort comprised 31 women (47.7\%; mean (SD) age 63.8 (12.6) years) and 34 men (52.3\%; 68.9 (8.0) years), and the older cohort comprised 17 women (54.8\%; 86.8 (4.3) years) and 14 male patients $(45.2 \% ; 84.3(3.6))$ years). There were no significant differences in sex distribution within (younger cohort $\mathrm{p}=0.80$; older cohort $\mathrm{p}=0.72$ ) or between cohorts $(p=0.51)$, or the sex averaged age within each cohort (younger cohort $p=0.16$; older cohort $p=0.08$ ). A significantly higher proportion of older patients had history of

\begin{tabular}{|c|c|c|}
\hline $\begin{array}{l}\text { Admission } \\
\text { NIHSS } \\
\text { score }\end{array}$ & Older, $n=31$ & $\begin{array}{l}\text { Younger, } \\
n=65\end{array}$ \\
\hline $\begin{array}{l}4-10 \\
\geqslant 11\end{array}$ & $\begin{array}{c}5(16.1 \%) \\
26(83.9 \%) \\
p=0.04, O R=\end{array}$ & $\begin{array}{l}24(36.9 \%) \\
41(63.1 \%) \\
\text { Cl } 1.03 \text { to } 8.98)\end{array}$ \\
\hline
\end{tabular}

congestive heart failure $(35.5 \% \quad v 7.7 \%, \mathrm{p}=0.001)$ or hypertension $(87.1 \%$ vs $66.2 \%, \mathrm{p}=0.03)$. In past medical history, the frequency of stroke or transient ischaemic attack, coronary heart disease, atrial fibrillation, diabetes, or antiplatelet or anticoagulant use was not significantly different between the two cohorts.

Mean (SD) onset to treatment time was 144.48 (28.21) minutes (range: 81 to 180 ) in the younger and 154.68 (23.66) minutes (range 105 to 187 ) in the older cohort $(\mathrm{p}=0.09)$. Only two patients received treatment under 90 minutes of stroke onset.

The average (SD) baseline stroke severity before receiving iv rt-PA as assessed by NIHSS was 13.7 (5.7) (range 6 to 27) in the younger cohort, and 15.4 (5.2) (range 6 to 25) in the older cohort. The difference between the averages of baseline stroke severity was not significant $(p=0.16, t$ test). Distribution of moderate (NIHSS score 4-10) and severe (NIHSS score $\geqslant 11$ ) baseline stroke between two cohorts is summarised in table 1. Significantly more patients in the older cohort presented with a more severe baseline stroke $(\mathrm{p}=0.04, \mathrm{OR}=3.04,95 \%$ CI 1.03 to 8.98$)$.

In hospital death caused by index stroke and its complications occurred in 6 of 65 patients $(9.2 \%)$ of the younger cohort, as opposed to 10 of $31(32.3 \%)$ of the older cohort $(\mathrm{p}=0.005 ; \mathrm{OR}=4.68 ; 95 \% \mathrm{CI} 1.52$ to 14.47$)$. In comparison to the younger cohort, patients in the older cohort were almost five times more likely to die from index stroke and its complications during hospitalisation $(\mathrm{p}=0.005$, $\mathrm{OR}=4.68$, CI 1.52 to 14.47) (table 2). Stroke related mortality remained significantly higher in the older cohort at 90 days follow up $(\mathrm{p}=0.01, \mathrm{OR}=3.95,95 \%$ CI 1.33 to 11.70$)$. Stroke related mortality at the end of 3 years' follow up was $15.4 \%$ in the younger and $48.4 \%$ in the older cohort $(\mathrm{p}=0.001, \mathrm{OR}=5.16$, $95 \%$ CI 1.95 to 13.67) (table 2). Kaplan-Meier estimates of survival rates up to 36 months after index stroke in both age cohorts is given in fig 1 , and demonstrates significant difference in overall survival $(\mathrm{pP}=0.003)$.

Regardless of baseline stroke severity, at 90 days follow up, 30 of 65 patients $(46.2 \%)$ in the younger cohort had NIHSS score of 0 or 1 , compared with 5 of 31 patients $(16.2 \%)$ in the older cohort $(\mathrm{p}=0.04, \mathrm{OR}=0.22 ; 95 \%$ CI 0.08 to 0.66$)$. At 90 days, 39 of 65 patients $(60.0 \%)$ in the younger and 5 of 31 patients $(16.1 \%)$ in the older cohort had BI of 95 or 100 $(\mathrm{p}<0.001, \mathrm{OR}=0.13 ; 95 \%$ CI 0.04 to 0.38$)$, while MRS of $0-2$ was attained in 38 of 65 (58.5\%) younger, and 5 of 31 (16.1\%) older patients $(\mathrm{p}<0.001$, OR $=0.14 ; 95 \%$ CI0.05 to 0.40$)$. Considering functional recovery, as measured by BI or MRS,

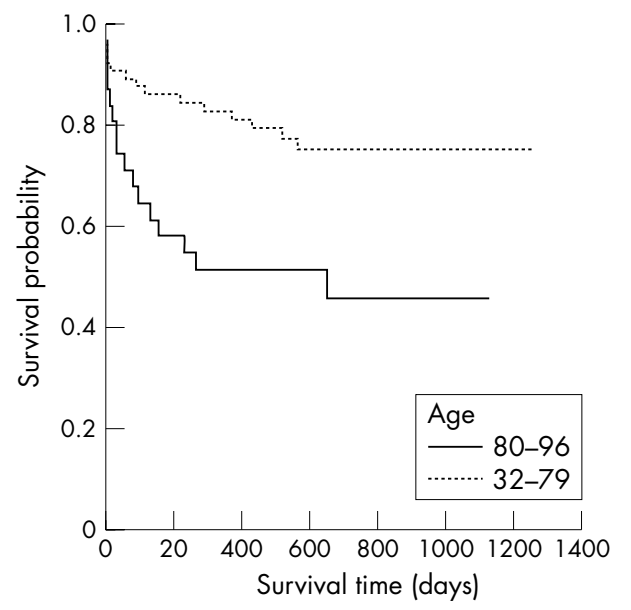

Figure 1 Kaplan-Meier estimates of the survival distribution for younger and older cohorts 
Table 2 Mortality at various follow up intervals

\begin{tabular}{|c|c|c|c|c|}
\hline \multirow[b]{2}{*}{ Mortality } & \multicolumn{2}{|l|}{ Age } & \multirow[b]{2}{*}{ Odds ratio $(95 \% \mathrm{Cl})$} & \multirow[b]{2}{*}{$p$ value } \\
\hline & $32-79$ & $80-96$ & & \\
\hline In hospital, stroke related & $6 / 65(9.2 \%)$ & $10 / 31$ (32.3\%) & 4.68 (1.52 to 14.47$)$ & 0.005 \\
\hline 90 days, stroke related & $7 / 65(10.8 \%)$ & $10 / 31$ (32.3\%) & $3.95(1.33$ to 11.70$)$ & 0.01 \\
\hline \multicolumn{5}{|l|}{ Up to 12 months } \\
\hline Stroke related & $8 / 65(12.3 \%)$ & $14 / 31(45.2 \%)$ & 5.87 (2.11 to 16.33 ) & $<0.001$ \\
\hline All causes & $11 / 65$ (16.9\%) & $15 / 31$ (48.4\%) & 4.60 (1.77 to 11.99$)$ & 0.001 \\
\hline \multicolumn{5}{|l|}{ Up to 3 years } \\
\hline Stroke related & $10 / 65$ (15.4\%) & $15 / 31(48.4 \%)$ & $5.16(1.95$ to 13.67$)$ & 0.001 \\
\hline All causes & $15 / 65(23.1 \%)$ & $16 / 31(51.5 \%)$ & 3.56 (1.43 to 8.84$)$ & 0.005 \\
\hline
\end{tabular}

older patients (regardless of baseline stroke severity) had only $13-14 \%$ the chance of their younger counterparts for independent survival.

When considering more severe baseline stroke (NIHSS score $\geqslant 11), 24$ of 26 patients $(92.3 \%)$ in the older cohort had poor outcome (dependent survival or stroke related death) at 90 day follow up, while poor outcome was seen in 22 out of 41 patients $(46.3 \%)$ in the younger cohort (table 3 ). Patients in the older cohort presenting with severe baseline stroke were 10.36 times more likely to have poor outcome compared with younger patients $(\mathrm{p}=0.001 ; \mathrm{OR}=10.4 ; 95 \%$ CI 2.16 to 49.20) (table 3). When baseline stroke was moderate, outcome did not significantly vary between younger and older cohorts $(\mathrm{p}=0.57$; OR 2.53; $95 \%$ CI 0.33 to 19.53$)$ (table 3).

When clinical outcomes were measured with the BI $(\leqslant 90$ indicating poor outcome) at 90 days, results were similar to MRS as indicated above. At 12 month follow up, clinical outcomes expressed as MRS and BI were also similar to those obtained at 90 days.

When all 96 patients were considered, there was no threshold age or baseline NIHSS score that predicted stroke related mortality, all mortality, follow up NIHSS score, BI, or MRS at any point up to 3 years.

Symptomatic haemorrhage occurred in 4/65 (6.2\%) patients in the younger cohort, and $3 / 31(9.7 \%)$ patients in the older cohort. The difference was not significant $(\mathrm{p}=0.69$, $\mathrm{OR}=1.63$, CI 0.34 to 7.79 ).

A multivariate logistic regression analysis was conducted to determine significant predictors of dependent survival indicated by MRS of $\geqslant 3$ at 90 days. Variables tested included age, gender, baseline stroke severity, hypertension, hyperglycaemia, hyperlipidaemia, intravenous treatment for hypertension or hyperglycaemia, coronary artery disease, and congestive heart failure. Of these, only baseline stroke severity and age were independent predictors for poor outcome (NIHSS score $\geqslant 11: p=0.001$, OR $=6.05,95 \%$ CI 2.08 to 17.55 ; age $\geqslant 80$ years: $p=0.001, \mathrm{OR}=6.42,95 \% \mathrm{CI}$ 2.04 to 20.14). As indicated by the ORs, baseline stroke severity and age were equal in predicting poor outcome.

\section{DISCUSSION}

In the NINDS trial there were only 42 patients of 80 years of age or older, but in clinical application there is no upper limit of age for iv rt-PA use in acute stroke. Several phase IV studies and the NINDS trial caution about less favourable outcome from iv rt-PA with advancing chronological age. The size and the statistical significance of the benefit from iv rt$\mathrm{PA}$ in the older cohorts (age $\geqslant 80$ years) versus their younger counterparts has not been clearly demonstrated. Although without thrombolytic treatment, advancing age alone predicts poor outcome from acute stroke, as does more severe stroke at the time of presentation, we are compelled to ask several questions: can the NINDS trial results be extrapolated to patients older than 80 years; are there thresholds for age or stroke severity predicting outcome; if we cannot demonstrate a threshold age or stroke severity what are the possible explanations; and is it possible that there are thresholds, but we have not yet recognised their surrogate markers?

Although in our study, age and baseline stroke severity were independent predictors of outcome, we found no threshold age or threshold baseline NIHSS score predicting outcome. This result is in agreement with NINDS trial and STARS study results and a recent non-thrombolytic therapy observational study. ${ }^{45-20}$ How can we explain these findings, where advancing chronological age indicates poor outcome from stroke but we cannot demonstrate a threshold age? Advancing chronological age crudely reflects the natural process of biological aging of the vascular system. If we assume that the combined risks of advancing age, genetic constitution, and modifiable risk factors of stroke determine the vascular age of the person, there will be a wide range of vascular ages among patients for a given chronological age, potentially eliminating the threshold effect of the latter.

Baseline NIHSS score may predict long term outcome..$^{21}$ Our and several other studies failed to demonstrate a threshold baseline NIHSS score predicting response to treatment. However, as the stroke severity increased odds for favourable outcome decreased. Expressing neurological deficit as a total score does not differentiate between qualitatively different and clinically more expressive deficits. A moderate sensory loss or a quadratic anopsia, or moderate slurring of speech is unlikely to cause dependent survival as measured by BI or MRS. Baseline NIHSS score overlooks the dynamic nature of brain embolism and the effect of collateral circulation on recovery from acute stroke with or without thrombolytic therapy. ${ }^{22}$ It has also been suggested that, compared with right hemispheric stroke, patients with left

Table 3 Admission NIHSS score versus MRS at 90 days in older and younger cohorts

\begin{tabular}{|c|c|c|c|c|}
\hline \multirow{2}{*}{$\begin{array}{l}\text { Age } \\
\text { (years) }\end{array}$} & \multicolumn{2}{|c|}{ NIHSS score 4-10* } & \multicolumn{2}{|c|}{ NIHSS score $\geqslant 11 \dagger$} \\
\hline & MRS $\geqslant 3$ & MRS: $0-2$ & MRS $\geqslant 3$ & MRS: 0-2 \\
\hline $\begin{array}{l}\geqslant 80 \\
\leqslant 79\end{array}$ & $2 / 5(40.0 \%)$ & $3 / 5(60.0 \%)$ & $24 / 26$ (92.3\%) & $2 / 26(7.7 \%)$ \\
\hline & $J /<4(20.8 \%)$ & $19 / 24(19.2 \%)$ & $22 / 41(33.6 \%)$ & $19 / 41$ (40.5\%) \\
\hline
\end{tabular}


hemispheric stroke may score five points higher in the NIHSS, and "for a given NIHSS score, the median volume of right hemisphere stroke is consistently larger than the median volume of a left hemisphere stroke".$^{23}$ The above factors potentially may obscure a threshold baseline NIHSS score predicting outcome. More specific surrogate markers may help with prognostication and increase the appropriateness of therapeutic choice. ${ }^{24}$

Because of the lack of placebo groups, and a relatively smaller number of patients, our study does not offer definitive evidence for withholding iv rt-PA in older patients when they present with severe stroke. However, our data indicate that advancing age and severe baseline stroke reflect less favourable odds for good recovery despite iv rt-PA. We inform patients (or next of kin) about our results prior to iv rt-PA. In particular, we indicate that the effect of iv rt-PA is yet to be demonstrated in a randomised trial to be significantly as effective in patients older than 80 years compared with younger patients. Considering that a severe stroke without thrombolytic therapy has a poor prognosis, especially in patients with advanced age, iv rt-PA may offer a guarded chance for recovery to independent survival in carefully selected older patients. However, more studies are needed to better understand the short and long term outcome of acute stroke in older patients treated with iv rt-PA, and better surrogate markers are needed for reliable prognostication.

\section{Authors' affiliations}

M S Mouradian, Department of Medicine, Division of Neurology, University of Alberta, Edmonton, Alberta, Canada

A Senthilselvan, Department of Public Health Sciences, University of Alberta, Edmonton, Alberta, Canada

G Jickling, University of Alberta, School of Medicine and Dentistry, University of Alberta, Edmonton, Alberta, Canadaa

J A McCombe, Division of Neurology, University of Alberta, University of Alberta, Edmonton, Alberta, Canada

D J Emery, Radiology and Diagnostic Imaging, University of Alberta, University of Alberta, Edmonton, Alberta, Canada

N Dean, Department of Medicine, Division of Neurology, University of Alberta, University of Alberta, Edmonton, Alberta, Canada

A Shuaib, Department of Medicine, Division of Neurology, University of Alberta, University of Alberta, Edmonton, Alberta, Canada

Competing interests: none declared

\section{REFERENCES}

1 The National Institute of Neurological Disorders and Stroke rt-PA Stroke Study group. Tissue plasminogen activator for acute ischemic stroke. New Engl J Med 1995:333:1581-7.

2 Kwiatkowski TG, Libman R, Frankel M, for the National Institute of Neurological Disorders and Stroke Recombinant Tissue Plasminogen Activator Stroke Study Group, et al. Effects of tissue plasminogen activator for acute ischemic stroke at one year. N Engl J Med 1999;340:1781-7.
3 Wardlaw JM. Sandercock PAG. Berge E. Thrombolytic therapy with recombinant tissue plasminogen activator for acute ischemic stroke. where do we go from here? a cumulative meta-analysis. Stroke 2003;34:1437-42.

4 The NINDS t-PA Stroke Study Group. Generalized efficacy of t-PA for acute stroke. Subgroup analysis of the NINDS t-PA Stroke Trial. Stroke 1997;28:2119-25.

5 Albers GW, Bates VE, Clark WM, et al. Intravenous tissue-type plasminogen activator for treatment of acute stroke. The Standard Treatment with Alteplase to Reverse Stroke (STARS) study. JAMA 2000;283:1145-50.

6 Tanne D, Gorman MJ, Bates VE, et al. Intravenous tissue plasminogen activator for acute ischemic stroke in patients aged 80 years and older. The tPA Stroke Survey experience. Stroke 2000;31:370-5.

7 Schlegel DJ, Tanne D, Demchuk AM, et al. Prediction of hospital disposition after thrombolysis for acute ischemic stroke using the National Institute of Health Stroke Scale. Arch of Neurol 2004;61:1061-4.

8 Norris JW, Buchan A, Cote R, et al. on behalf of The Canadian Stroke Consortium. Canadian guidelines for intravenous thrombolytic treatment in acute stroke. Can J Neurol Sci 1998;25:257-9.

9 Adams HP, Brott TG, Furlan AJ, et al. Guidelines for thrombolytic therapy for acute stroke: A supplement to the guidelines for the management of patients with acute ischemic stroke. A statement for healthcare professionals from a special writing group of the Stroke Council, American Heart Association. Stroke 1996;27:1711-18.

10 Lyden P, Brott T, Tilley B, et al. Improved reliability of the NIH Stroke Scale using video training. Stroke 1994;25:2220-6.

11 van Swieten JC, Koudstaal PJ, Visser MC, et al. Inter-observer agreement for the assessment of handicap in stroke patients. Stroke 1988;19:604-7.

12 Mahoney FI, Barthel DW. Functional evaluation: the Barthel index. Md Med J 1965;14:61-5.

13 Hacke W, Kaste M, Fieschi C, et al. for the Second European-Australasian Acute Stroke Study Investigators. Randomised double-blind placebocontrolled trial of thrombolytic therapy with intravenous alteplase in acute ischeminc stroke (ECASS II). Lancet 1998;352:1245-51.

14 Fiorelli M, Bastianello S, von Kummer R, et al. Hemorrhagic transformation within 36 hours of a cerebral infarct: relationships with early clinical deterioration and 3-month outcome in the European Cooperative Acute Stroke Study I (ECASS I) cohort. Stroke 1999;30:2280-4.

15 Berger C, Fiorelli M, Steiner T, et al. Hemorrhagic transformation of ischemic brain tissue. Asymptomatic or symptomatic? Stroke 2001;32:1330-5.

16 Frankel MR, Morgenstern LB, Kwiatkowski T, et al. For the National Institute of Neurological Disorders and Stroke rt-PA Stroke Study Group. Predicting prognosis after stroke. A placebo group analysis from the National Institute of Neurological Disorders and Stroke rt-PA Stroke trial. Neurology 2000;55:952-9.

17 Chambers BR, Norris JW, Shurvell BL, et al. Prognosis of acute stroke. Neurology 1987;37:221-5.

18 Censori B, Camerlingo M, Casto L, et al. Prognostic factors in first-ever stroke in the carotid artery territory seen within 6 hours after onset. Stroke 1993;24:532-5.

19 Henon H, Godefroy O, Leys D, et al. Early predictors of death and disability after acute cerebral ischemic event. Stroke 1995;26:392-8.

20 Appelros P, Nydevik I, Viitanen M. Poor outcome after first-ever stroke. Predictors for death, and recurrent stroke within the first year. Stroke 2003;34:122-6.

21 Adams HP Jr, Davis PH, Leira EC, et al. Baseline NIH Stroke Scale score strongly predicts outcome after stroke: a report of the Trial of Org 10172 in Acute Stroke Treatment (TOAST). Neurology 1999:53:126-31.

22 Babikian VL, Caplan LR. Brain embolism is a dynamic process with variable characteristics. Neurology 2000;54:797-801.

23 Woo D, Broderick JP, Kothari RU, et al, for the NINDS t-PA Stroke Study Group. Does the National Institute of Health Stroke Scale favor left hemisphere strokes? Stroke 1999;30:2355-9.

24 Buchan AM, Barber PA, Newcommon N, et al. Effectiveness of t-PA in acute ischemic stroke: outcome relates to appropriateness. Neurology 2000;54:679-84. 